\title{
A Classifier For Ideal Unimodular Singularities
}

\author{
Muhammad Ahsan Binyamin, Junaid Alam Khan and \\ Hasan Mahmood
}

\begin{abstract}
In this article we characterize the ideal unimodular singularities in terms of their invariants. On the basis of this characterization we give an implementation of a classifier for ideal unimodular singularities in the computer algebra system SINGULAR.
\end{abstract}

\section{Introduction}

In [1] Arnold, V. I. and in [10] Schappert, A. classified the unimodular plane curve singularities with respect to right and contact equivalence respectively. And then Drozd and Greuel in [5] introduced the notion of ideal-unimodal plane curve singularities (IUS). In characteristic zero ideal-unimodal singularities and contact unimodal singularities coincide. Nguyen Hong Duc in [6] introduced some invariants for IUS and also gave pre-normal forms of all IUS by using the theorem on parametrization finite determinacy.

In this article we will give a sufficient condition for a plane curve singularity to be an IUS in terms of certain invariants and also give the implementation of a classifier for IUS in the computer algebra system SINGULAR [4], [8].

The classifier is based on the computation of several invariants. This computation turns out to be difficult for reducible curves. Therefore we show in section-2 how to reduce this computations to compute the corresponding invariants for the branches. This turn out to be much more efficient.

Key Words: Ideal unimodular singularities, Conductor, Intersection multiplicity

2010 Mathematics Subject Classification: Primary 14B05; Secondary 14H20.

Received: December, 2013.

Revised: January, 2014.

Accepted: January, 2014. 


\section{2 computation of invariants}

The aim of this section is to present an algorithm which reduces the computation of the delta invariant and the conductor of a reduced plane curve singularity to that of their irreducible branches. We want to compare the two different approaches to compute the delta invariant of any reduced plane curve singularity. From the computational point of view, one approach computes the delta invariant by using the Humburger-Noether expansion of a reduced plane curve singularity and our new approach computes the delta invariant of a reduced plane curve singularity by using the Humburger-Noether expansion of the irreducible components. For details see [2]. Examples show that our approach is much faster (see section-4).

Remark 2.1. We will compare our procedure with the procedure delta from the SINGULAR library hnoether.lib.

Let $\mathbf{R}$ be a plane curve singularity, $\mathbf{m}$ its maximal ideal and $\mathbf{R}_{\mathbf{0}}$ be its integral closure. It follows from [7], [3] that $\mathbf{R}_{\mathbf{0}} \cong \oplus_{i=1}^{r} K[[t]]$, where $\mathrm{r}$ is the number of branches of $f$. Then the following definitions can be found in [6].

Definition 2.2. Let $0 \neq f \in \mathbf{m} \subset \mathbf{K}[[x, y]]$ be reduced and $\mathbf{R}=\mathbf{K}[[x, y]] /<$ $f>\hookrightarrow \mathbf{R}_{\mathbf{0}}$ be its normalization. Then a composition $\psi$ of the natural projection $\mathbf{K}[[x, y]] \rightarrow \mathbf{R}$, the normalization $\mathbf{R} \hookrightarrow \mathbf{R}_{\mathbf{0}}$ and an isomorphism $\mathbf{R}_{\mathbf{0}} \cong \oplus_{i=1}^{r} K[[t]]$ is called parametrization of $f$.

Definition 2.3. Let $f \in \mathbf{K}[[x, y]]$ be reduced and $f_{i}$ the irreducible components of $f$. Also let $\psi$ be a parametrization of $f$.

1. We call $\delta(f)=\operatorname{dim}_{\mathbf{K}} \mathbf{R} / \mathbf{R}_{\mathbf{0}}$ the $\delta$-invariant of $f$.

2 . We introduce the valuation maps

$$
v=\left(v_{1}, \ldots, v_{r}\right): \mathbf{R} \rightarrow\left(\mathbb{Z}_{\geq 0} \cup \infty\right)^{r}
$$

defined by

$$
g \mapsto \operatorname{ord}\left(g\left(x_{i}(t), y_{i}(t)\right)\right)_{i=1, \ldots, r},
$$

where $\left(x_{i}(t), y_{i}(t)\right)_{i=1, \ldots, r}$ is the parametrization of each irreducible branch of $f$. Its image $\mathbf{S}(f)=v(\mathbf{R})$ is a semigroup.

3. Let $\mathcal{C}=\left(\mathbf{R}: \mathbf{R}_{\mathbf{0}}\right)=\left\{u \in \mathbf{R}: u \mathbf{R}_{\mathbf{0}} \subset \mathbf{R}\right\}$ be the conductor ideal of $\mathbf{R}$ in $\mathbf{R}_{\mathbf{0}}$ of the form $\left\langle\left(t_{1}^{c_{1}}, \ldots, t_{n}^{c_{n}}\right)>\right.$ (see- [11]). We call $\mathbf{c}(f)=\left(c_{1}, \ldots, c_{n}\right)$ the conductor, $\underline{\mathbf{m t}}(f)=\left(\boldsymbol{\operatorname { m t }}\left(f_{1}\right), \ldots, \boldsymbol{m t}\left(f_{n}\right)\right)$ the multi-multiplicity and,$\underline{\mathbf{c}}(f)=$ $\left(c\left(f_{1}\right), \ldots, c\left(f_{n}\right)\right)$ the multi-conductor of $f$, where $c\left(f_{i}\right)$ is the conductor of i-th component of $f$.

Definition 2.4. 1. Let $g \in \mathbf{K}[[x, y]]$ be irreducible and $(x(t), y(t))$ a parametrization. Then intersection multiplicity of any $f \in \mathbf{K}[[x, y]]$ with $g$ 
is given by

$$
i(f, g)=\operatorname{ord}(f(x(t), y(t))) .
$$

If $u$ is a unit then we define $i(f, u)=0$.

2. The intersection multiplicity with reducible $g=g_{1}, \ldots, g_{s}$, where $g_{i}$ are irreducible is defined by the following sum

$$
i(f, g)=i\left(f, g_{1}\right)+\cdots+i\left(f, g_{s}\right) .
$$

The following propositions give the conductor and delta invariant in terms of irreducible branches and their intersection multiplicities.

Proposition 2.5. Let $f=f_{1} \ldots f_{r}$ with $f_{i}$ irreducible, then

$$
\mathbf{c}(f)=\left(c\left(f_{1}\right)+\sum_{j \neq 1} i\left(f_{1}, f_{j}\right), \ldots, c\left(f_{r}\right)+\sum_{j \neq r} i\left(f_{r}, f_{j}\right)\right)
$$

In particular if $f$ is irreducible then $\mathbf{c}(f)=2 \delta(f)$.

Proof. See [9].

Proposition 2.6. Let $f=f_{1} \ldots f_{r}$ with $f_{i}$ irreducible, then

$$
\delta(f)=\sum_{i=1}^{r}\left(\delta\left(f_{i}\right)+\sum_{j \neq i} i\left(f_{i}, f_{j}\right)\right)
$$

Proof. See [6].

\section{The Algorithm}

In this section we present the algorithm to compute delta invariant, conductor, multi-conductor, number of branches and multi-mutiplicity of any plane curve singularity $f$. This algorithm has been implemented in SINGULAR ([8],[4]).

\section{Timings and Table}

In this section we provide some examples and a table which gives a time comparison between the algorithm delta (already implemented in SINGULAR) and our algorithm redInvariant described above.Timings are conducted by using Singular 3-1-3 on an Intel@T2400, dual core $1.83 \mathrm{GHz}$ processor, $1 \mathrm{~GB}$ RAM under the Window 2007 operating system. 
Algorithm 1 Computing delta invariant, conductor, multi-conductor, number of branches and multi-mutiplicity of the reduced plane curve singularity (redInvariant)

Require: a polynomial $f,(f$ defines a reduced curve singularity).

Ensure: a list L containing the delta invariant, conductor, multi-conductor, number of branches and multi-mutiplicity of the reduced plane curve singularity.

1: factorize $f=f_{1}, \ldots, f_{r}$ (In SINGULAR this can be done by using the procedure factorize $(f)$.).

2: compute a list $H$, the Hamburger-Noether expansion (In SINGULAR this can be done by using the procedure hnexpansion $\left(f_{i}\right)$ from hnoether.lib .) for all $f_{i}$.

3: compute the matrix of intersection multiplicities (if $h_{1}, h_{2}$ is the output of hnexpansion then in SINGULAR the procedure intersection $\left(h_{1}, h_{2}\right)$ computes the intersection multiplicity of the branches corresponding to $h_{1}$ and $h_{2}$.) corresponding to the irreducible branches.

4: compute delta invariant as described in proposition-2.6.

5: compute conductor of $f$ as described in proposition-2.5.

6: compute parametrization (if $h$ is the output of hnexpansion then in SINGULAR the procedure param $(h)$ computes the parametrization of the branch corresponding to $h$.) of each branch of $f$

7: compute multi-conductor and multi-multiplicity as defined in definition2.3 .

8: add delta invariant, conductor, multi-conductor and multi-multiplicity to the list L.

9: return $\mathrm{L}$. 
We consider the following polynomials:

$$
\begin{aligned}
f_{1}= & \left(\left(x^{7}-y^{6}\right)^{4}-x^{20} y^{10}+x^{35}\right) \cdot\left(\left(x^{7}-y^{6}\right)^{3}-x^{24}+y^{25}\right) \cdot\left(\left(x^{7}-y^{5}\right)^{3}-x^{24}-y^{25}\right) \\
f_{2}= & \left(x^{14}+x^{5} y^{5}+y^{10}+y^{15}\right) \cdot\left(x^{16}+x^{18}+y^{20}+y^{23}\right) \\
f_{3}= & \left(x^{14}-y^{12}+x^{10} y^{4}+y^{19}\right) \cdot\left(x^{12}-y^{14}+x^{15}+y^{15}\right) \cdot\left(\left(x^{6}-y^{5}\right)^{4}-x^{26} y^{12}+\right. \\
& \left.x^{28}+y^{30}\right) \cdot\left(x^{14}+y^{18}+y^{21}\right) \\
f_{4}= & \left(x^{8}+2 y^{14}\right) \cdot\left(x^{10}+5 y^{10}\right) \cdot\left(y^{2}-x^{3}\right) \cdot\left(x^{2}-y^{3}\right) \\
f_{5}= & \left(x^{18}+y^{24}+x^{29}\right) \cdot\left(x^{14}+y^{18}+y^{21}\right) \cdot\left(x^{9}-x^{3} y^{3}+y^{11}\right) \cdot\left(x^{15}-y^{10}-y^{19}\right) \\
f_{6}= & \left(x^{16}+3 y^{18}\right) \cdot\left(x^{5}+7 y^{5}\right) \\
f_{7}= & \left(\left(x^{7}-y^{6}\right)^{4}-x^{20} y^{10}+x^{35}\right) \cdot\left(\left(x^{7}-y^{6}\right)^{3}-x^{24}+y^{25}\right) \\
f_{8}= & \left(x^{18}+y^{24}+x^{29}\right) \cdot\left(x^{14}+y^{18}+y^{21}\right) \cdot\left(x^{9}-x^{3} y^{3}+y^{11}\right)
\end{aligned}
$$

\section{Table 1}

\begin{tabular}{|c|c|c|}
\hline Poly & redInvariant & delta \\
\hline$f_{1}$ & $10 \mathrm{sec}$ & $25 \mathrm{sec}$ \\
\hline$f_{2}$ & $2 \mathrm{sec}$ & $>1 \mathrm{hr}$ \\
\hline$f_{3}$ & $15 \mathrm{sec}$ & $5 \mathrm{~min}$ \\
\hline$f_{4}$ & $5 \mathrm{~min}$ & $>1 \mathrm{hr}$ \\
\hline$f_{5}$ & $10 \mathrm{sec}$ & $>1 \mathrm{hr}$ \\
\hline$f_{6}$ & $2 \mathrm{sec}$ & $>1 \mathrm{hr}$ \\
\hline$f_{7}$ & $8 \mathrm{sec}$ & $3 \mathrm{sec}$ \\
\hline$f_{8}$ & $8 \mathrm{sec}$ & $25 \mathrm{sec}$ \\
\hline
\end{tabular}

\section{Drozd-Greuel's Ideal-Unimodal Plane Curve Singularities}

The following definition can be found in [5].

Definition 5.1. A plane curve singularity with complete local ring $\mathbf{R} \subset \mathbf{R}_{\mathbf{0}}$ is called ideal-unimodal plane curve singularity (IUS) if its maximal ideal admits generators $x, y$ whose valuation satisfies the condition in Table-2. Moreover we say that $\mathbf{R}$ is of type $\mathbf{E}, \mathbf{T}, \mathbf{W}$ and $\mathbf{Z}$, if $\mathbf{R}$ belongs to the corresponding class. 


Table 2
\begin{tabular}{c|c|c|c|c|l|}
\hline Type & $\mathrm{r}$ & $v(x)$ & $v(y)$ & Condition & Name \\
\hline \multirow{2}{*}{$E$} & 1 & $(3)$ & $(l)$ & $l=7,8,10,11$ & $E_{2 l-2}$ \\
& 2 & $(1,2)$ & $(\infty, l)$ & $l=4,5,6,7$ & $E_{2,2 q-1}, E_{13}$, \\
& & $(1,1,1)$ & $(\infty, l, k)$ & $l=2,3, k \geq l$ & $E_{3,2 q-1}, E_{19},(q \geq 1)$ \\
& 3 & $E_{l, 2(k-1)}$ \\
\hline & 2 & $(2, k)$ & $(l, 2)$ & $l, k$ odd, $l k>4$ & $T_{k+2, l+2,2}$ \\
$T$ & 3 & $(1,1, k)$ & $(\infty, l, 2)$ & $k$ odd, $l k \geq 2$ & $T_{k+2,2 l+2,2}$ \\
& 4 & $(1, \infty, 1, k)$ & $(\infty, 1, l, 1)$ & $l k \geq 1$ & $T_{2 k+2,2 l+2,2}$ \\
\hline & 1 & $(4)$ & $(l)$ & $l=5,6,7\left(^{*}\right)$ & $W_{12}, W_{1,2 q-1}^{\sharp}$, \\
& & & & & $W_{18},(q \geq 1)$ \\
$W$ & 2 & $(1,3)$ & $(\infty, l)$ & $l=4,5$ & $W_{13}, W_{17}$ \\
& 2 & $(2,2)$ & $(3, l)$ & $l=3, l \geq 5$ odd $\left(^{*}\right)$ & $W_{1,0}, W_{1,2 q}^{\sharp}$, \\
& & & & & $W_{1, l-3},(q \geq 1)$ \\
& 3 & $(1,1,2)$ & $(\infty, l, 3)$ & $l \geq 2$ & $W_{1,2 l-3}$ \\
\hline & 2 & $(1, l)$ & $(\infty, 3)$ & $l=4,5,7,8$ & $Z_{11}, Z_{13}, Z_{17}, Z_{19}$ \\
$Z$ & 3 & $(1, \infty, 2)$ & $(\infty, 1, l)$ & $l=2,3,4,5$ & $Z_{0,2 q-1}, Z_{12}, Z_{1,2 q-1}$, \\
& & & & & $Z_{18},(q \geq 1)$ \\
& 4 & $(1, \infty, 1,1)$ & $(\infty, 1, l, k)$ & $l=1,2, k \geq l$ & $Z_{l-1,2(k-1)}$ \\
\hline
\end{tabular}

In Table-2, $r$ denotes the number of branches and $v(x), v(y)$ denote the valuation of $x$ and $y$ respectively.

$\left.{ }^{*}\right)$ If $\operatorname{char}(\mathbf{K})=2$, extra conditions in case $W$ are required: if $r=1$ then $l=5$, if $r=2, v(x)=(2,2)$ and $l=3$ then $x^{2}-y^{3} \notin t^{7} \mathbf{R}_{\mathbf{0}}$.

(This excludes $W_{18}(r=1)$ and all $W_{1, q}^{\sharp}(r=1)$ or $\left.(r=2)\right)$. For details see [5], [6].

Remark 5.2. The index $q$ depends on the delta invariant of the corresponding singularity. The relation between $q$ and the delta invariant of the corresponding singularity is given in [6].

The following proposition indicates the overlapped classes of ideal-unimodal singularities given in Table-2.

Proposition 5.3. The following classes of singularities coincide.

(i) $E_{2,2 q-1}=T_{2 q+3,3,2} ; q \geq 1 ;(r=2)$.

(ii) $E_{2,2(l-1)}=T_{3,3 l+2,2} ; l \geq 2 ;(r=3)$.

(iii) $T_{k+2, l+2,2}=T_{l+2, k+2,2}$ and $T_{2 k+2,2 l+2,2}=T_{2 l+2,2 k+2,2}$.

(iv) $Z_{0,2 q-1}=T_{2 q+3,4,2} ; q \geq 1 ;(r=3)$.

(v) $Z_{0,2(k-1)}=T_{2 k+2,4,2} ; k \geq 1 ;(r=4)$.

Proof. See [6].

The following proposition gives us the several invariants of ideal-unimodal singularities. 
Proposition 5.4. The conductor (c), multi-multiplicity ( $\underline{\mathbf{m t}})$ and the multiconductor $(\underline{\mathbf{c}})$ of the ideal-unimodal singularities are given in the Table-3.

Proof. See [6].

The following definition and lemma can be found in [3].

Definition 5.5. Let $f \in \mathbf{K}[[x, y]]$ be reduced and $f_{i}$ the irreducible components of $f$. Let $\gamma \in \mathbf{K}[[x, y]]$ be regular. Then we call $\bar{\beta}_{1}(f)=\sup \{\min$ $i\left(f_{i}, \gamma\right): \gamma$ is regular $\}$ the maximal contact multiplicity of $f$.

Lemma 5.6. Let $f \in K[[x, y]]$ be irreducible s.t. $m=m t(f)=\operatorname{ord} f(0, y)$ and let $n=\operatorname{ord} f(x, 0)$. Then

1. If $f$ is non-singular, then there exist a coordinate change $\Phi \in A u t_{K} K[[x, y]]$ such that $\Phi(f)(x, y)=y$.

2. If $f$ is singular, then there exist a coordinate change $\Phi \in A u t_{K} K[[x, y]]$ of the form

$$
\Phi(x)=x+c_{1} y^{d_{1}}+c_{2} y^{d_{2}}+\ldots
$$

and

$$
\Phi(y)=y
$$

such that $m \nmid k$, where $k=\operatorname{ord} \Phi(f)(x, 0)$. In particular, if $m / n$ then $c_{1} \neq 0$ and $d_{1}=\frac{n}{m}$. If $m \nmid n$ then $c_{i}=0$ for all $i \geq 1$. Moreover $\bar{\beta}_{1}(f)=k$.

Following corollary and lemma can be found in [6].

Corollary 5.7. Let char $(K) \neq 2$ and let $f \in K[[x, y]]$ be irreducible with $m t(f)=2$ and $\delta(f)=k \geq 1$. Then $f$ is contact equivalent to $x^{2 k+1}-y^{2}$.

Lemma 5.8. Let $f$ is an irreducible plane curve singularity having a parametrization $(x(t), y(t))$ with $\operatorname{ord} x(t)=n$, ordy $(t)=m$ and $\operatorname{gcd}(m, n)=1$. Then

$$
\mathbf{c}(f)=(m-1)(n-1) .
$$

\section{Characterization of ideal-unimodal singularities in terms of their invariants}

In this section we give the sufficient condition for a plane curve singularity to be an ideal-unimodal singularity in terms of its branches, multi-multiplicity, multi-conductor and the conductor.

Lemma 6.1. Let $f \in K[[x, y]]$ such that $f=f_{1} f_{2}$ and $m t\left(f_{1}\right)=2=m t\left(f_{2}\right)$, where each $f_{i}$ is irreducible. Assume that $\mu\left(f_{1}\right) \leq \mu\left(f_{2}\right)$ and $i\left(f_{1}, f_{2}\right)<\infty$. If the tangent cones of $f_{1}$ and $f_{2}$ are different then $i\left(f_{1}, f_{2}\right)=4$ otherwise $i\left(f_{1}, f_{2}\right) \geq 2\left(\mu\left(f_{1}\right)+1\right)$. 
Proof. We may assume that $f_{1}=x^{2}-y^{l}$ and $f_{2}=(\alpha x+\beta y)^{2}+$ terms of degree $\geq 2$. If $\beta \neq 0$ then $i\left(f_{1}, f_{2}\right)=4$. If $\beta=0$ then we may assume that $\alpha=1$. We may write $f_{2}=x^{2}(1+$ terms of degree $\geq 1)+x\left(\gamma y^{s}+\right.$ terms of degree $\geq s)+y^{k}(\delta+$ terms of degree $\geq 1), \delta \neq 0$ because $f_{2}$ is irreducible. Since by assumption $\mu\left(f_{1}\right) \leq \mu\left(f_{2}\right)$ we obtain $k \geq l$ and $2 s \geq l$. This implies $i\left(f_{1}, f_{2}\right)=f_{2}\left(t^{l}, t^{2}\right) \geq 2 l=2\left(\mu\left(f_{1}\right)+1\right)$.

Lemma 6.2. Let $f \in K[[x, y]]$ be irreducible such that $m t(f)=4$.

1- If $\tau(f)=18=\mu(f)$ then $f \in W_{18}$

2- If $\tau(f)=16<\mu(f)=18$ then $f \in W_{1,3}^{\sharp}$.

Proof. We may assume that $x(t)=t^{4}+$ higher degree terms and $y(t)=t^{a}+$ higher degree terms, $a>4$ and $4 \nmid a$.

1- If $a$ is odd then lemma-5.8 gives $v(x)=4$ and $v(y)=7$, this implies $f \in W_{18}$. 2- If $a$ is even then the characteristic exponents are $k_{0}=4, k_{1}=a$ and $k_{2}=$ some odd number. This implies $\mu(f)=k_{0}+k_{1}+k_{2}-1$. As $a \geq 6$ this implies $a=6$ and $k_{2}=9$. So we obtain a singularity parametrized by $x=t^{4}$ , $y=t^{6}+t^{9}$, which gives $f \in W_{1,3}^{\sharp}$.

Proposition 6.3. A singularity $f \in K[[x, y]]$ is an ideal-unimodal singularity if and only if its number of branches, its multi-multiplicity ( conductor $(\underline{c})$ and its conductor $(\mathbf{c})$ are given as in the Table-3.

Proof. If $f$ is an ideal-unimodal singularity then its number of branches, its multi-multiplicity ( $\underline{\mathbf{m t}})$, its multi-conductor $(\underline{c})$ and its conductor $(\mathbf{c})$ are given by Proposition-5.4.

Now let $f \in K[[x, y]]$ having number of branches, multi-multiplicity, multiconductor and the conductor as given in Table-3, then we need to show that $f$ is an ideal-unimodal singularity.

For the class $E_{2 l-2}$ : Let $f \in K[[x, y]]$ be such that $r=1, \underline{\mathbf{m t}}=(3), \underline{c}=$ $(2 l-2), \mathbf{c}=(2 l-2)$ and $p \neq 2$. Since $\operatorname{gcd}(3, l)=1$ then lemma-5.8 gives $v(x)=3$ and $v(y)=l$ and hence $f$ is an ideal-unimodal singularity of type $E_{2 l-2}$.

The proof for the classes $W_{12}$ and $W_{18}$ is similar to the proof of the class $E_{2 l-2}$.

For the class $E_{3,2(k-1)}$ : Let $f \in K[[x, y]]$ be such that $r=3, \underline{\mathbf{m t}}=(1,1,1)$, $\underline{c}=(0,0,0), \mathbf{c}=(k+3,6, k+3)$ and $p \neq 2$. Then $f=f_{1} \cdot f_{2} \cdot f_{3}$ with $m t\left(f_{1}\right)=m t\left(f_{2}\right)=m t\left(f_{3}\right)=1$. By a suitable coordinate change we may assume that $f_{1}=y$. By using proposition-2.5 we can deduce that $i\left(f_{1}, f_{2}\right)=3$, $i\left(f_{2}, f_{3}\right)=3$ and $i\left(f_{1}, f_{3}\right)=k$. This implies that $i\left(f_{1}, y\right)=\infty, i\left(f_{2}, y\right)=3$ and $i\left(f_{3}, y\right)=k$ and therefore $i\left(f_{1}, x\right)=1, i\left(f_{2}, x\right)=1$ and $i\left(f_{3}, x\right)=1$ since $m t\left(f_{1}\right)=m t\left(f_{2}\right)=m t\left(f_{3}\right)=1$. It yields $v(x)=(1,1,1)$ and $v(y)=(\infty, 3, k)$ 
and hence $f \in E_{3,2(k-1)}$.

The proof for the classes $E_{13}, E_{19}, E_{3,2 q-1}, T_{k+2,2 l+2,2}, T_{2 k+2,2 l+2,2}, W_{13}$, $W_{17}, W_{1,2 l-3}, Z_{2 l+3}, Z_{12}, Z_{18}, Z_{1,2 q-1}$ and $Z_{1,2(k-1)}$ is similar to the proof of the class $E_{3,2(k-1)}$.

For the class $T_{k+2, l+2,2}$ : Let $f \in K[[x, y]]$ be such that $r=2$, $\underline{\mathbf{m t}}=(2,2)$, $\underline{c}=(l-1, k-1), \mathbf{c}=(l+3, k+3)$. Then $f=f_{1} \cdot f_{2}$ with $m t\left(f_{1}\right)=2$ and $m t\left(f_{2}\right)=2$. We need to show that $v(x)=(2, k)$ and $v(y)=(l, 2)$. If $p \neq 2$ then corollary-5.7 gives $f_{1}$ is contact equivalent to $x^{2}-y^{l}$ and $f_{2}$ is contact equivalent to $x^{2}-y^{k}$. Let $\varphi$ be an automorphism such that

$$
\varphi\left(f_{1}\right)=x^{2}-y^{l} .
$$

We may assume $f_{1}=x^{2}-y^{l}$ and $f_{2}=(\alpha x+\beta y)^{2}+$ higher degree terms. Now if $f_{1}$ and $f_{2}$ have different tangent cones then lemma-6.1 implies $i\left(f_{1}, f_{2}\right)=4$ and then proposition-2.5 gives $\mathbf{c}=(l+3, k+3)$ and this implies $v(x)=(2, k)$ and $v(y)=(l, 2)$. So $f \in T_{k+2, l+2,2}$. And for the other case $\mathbf{c} \neq(l+3, k+3)$. The proof for the classes $W_{1,0}, W_{1,2 q}^{\sharp}, W_{1,2 q-1}^{\sharp}$ and $W_{1, l-3}$, is similar to the proof of the class $T_{k+2, l+2,2}$.

Remark 6.4. To distinguish the classes $W_{18}$ and $W_{1,3}^{\sharp}$ we need one more invariant which is Tjurina number (see lemma-6.2).

\section{Short Description of the Classifier}

The formal algorithm of our classifier takes too much space, so we describe it shortly in the following two steps.

- It computes the number of branches, delta invariant, multi-multiplicity, multi-conductor and conductor of the given plane curve singularity (Algorithm-1).

- It compares the invariants of a given plane curve singularity with the invariants given in table-3 and if the given plane curve singularity is IUS then it returns to which class it belongs. 


\begin{tabular}{|c|c|c|c|c|c|c|}
\hline \multicolumn{7}{|l|}{ Table 3} \\
\hline Name & $\mathrm{r}$ & Condition & $\underline{\mathbf{m t}}$ & $\underline{\mathbf{c}}$ & $\mathbf{c}$ & $\begin{array}{l}\operatorname{char}(K) \\
=p\end{array}$ \\
\hline$E_{2 l-2}$ & 1 & $l=7,8,10,11$ & (3) & $(2 l-2)$ & $(2 l-2)$ & $p \neq 2$ \\
\hline$E_{13}, E_{19}$ & 2 & $l=5,7$ & $(1,2)$ & $(0, l-1)$ & $(l, 2 l-1)$ & - \\
\hline$E_{3,2 q-1}$ & 2 & - & $(1,2)$ & $(0,2 q+4)$ & $(6,2 q+10)$ & - \\
\hline$E_{3,2(k-1)}$ & 3 & $k \geq 3$ & $(1,1,1)$ & $(0,0,0)$ & $\begin{array}{l}(k+3,6, k+ \\
3)\end{array}$ & $p \neq 2$ \\
\hline$T_{k+2, l+2,2}$ & 2 & $\begin{array}{l}k \geq l \text { odd, } l k> \\
4\end{array}$ & $(2,2)$ & $(l-1, k-1)$ & $(l+3, k+3)$ & $p \neq 2$ \\
\hline$T_{k+2,2 l+2,2}$ & 3 & $k$ odd, $l k \geq 2$ & $(1,1,2)$ & $(0,0, k-1)$ & $\begin{array}{l}(l+2, l+ \\
2, k+3)\end{array}$ & $p \neq 2$ \\
\hline$T_{2 k+2,2 l+2,2}$ & 4 & $k \geq l \geq 1$ & $(1,1,1,1)$ & $(0,0,0,0)$ & $\begin{array}{l}(l+2, k+ \\
2, l+2, k+2) \\
\end{array}$ & - \\
\hline$W_{12}$ & 1 & $l=5$ & (4) & $(3 l-3)$ & $(3 l-3)$ & $p \neq 3$ \\
\hline$W_{18}$ & 1 & $l=7$ & (4) & $(3 l-3)$ & $(3 l-3)$ & $p \neq 2,3$ \\
\hline$W_{1,2 q-1}^{\sharp}$ & 1 & - & (4) & $(2 q+14)$ & $(2 q+14)$ & $p \neq 2$ \\
\hline$W_{13}, W_{17}$ & 2 & $l=4,5$ & $(1,3)$ & $(0,2 l-2)$ & $(l, 3 l-2)$ & - \\
\hline$W_{1,0}$ & 2 & - & $(2,2)$ & $(2,2)$ & $(8,8)$ & $p \neq 2$ \\
\hline$W_{1,2 q}^{\sharp}$ & 2 & - & $(2,2)$ & $(2,2)$ & $(q+8, q+8)$ & $p \neq 2$ \\
\hline$W_{1, l-3}$ & 2 & $l \geq 5$ odd & $(2,2)$ & $(2, l-1)$ & $(8, l+5)$ & $p \neq 2$ \\
\hline$W_{1,2 l-3}$ & 3 & $l \geq 2$ & $(1,1,2)$ & $(0,0,2)$ & $\begin{array}{l}(l+3, l+ \\
3,8)\end{array}$ & $p \neq 2$ \\
\hline$Z_{2 l+3}$ & 2 & $l=4,5,7,8$ & $(1,3)$ & $(0,2 l-2)$ & $(3,2 l+1)$ & - \\
\hline$Z_{12}, Z_{18}$ & 3 & $l=3,5$ & $(1,1,2)$ & $(0,0, l-1)$ & $\begin{array}{l}(l+1,3,2 l+ \\
1)\end{array}$ & $p \neq 2$ \\
\hline$Z_{1,2 q-1}$ & 3 & - & $(1,1,2)$ & $(0,0,2 q+2)$ & $(5,3,2 q+8)$ & $p \neq 2$ \\
\hline$Z_{1,2(k-1)}$ & 4 & $k \geq 2$ & $(1,1,1,1)$ & $(0,0,0,0)$ & $\begin{array}{lr}(k & + \\
3,3,5, k+3)\end{array}$ & 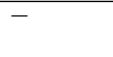 \\
\hline
\end{tabular}

Acknowledgements We would like to thank Dr. Gerhard Pfister and Dr. Nguyen Hong Duc for useful conversations.

\section{References}

[1] Arnold, V. I.; Classification of unimodal critical points of functions, Funct. Anal. App. 7(1973), 230-231.

[2] Binyamin, M. A.; Improving the computation of invariants of plane curve singularities, An. St. Univ. Ovidius Constanta, Vol. 21(1), 2013, 51-58.

[3] Campillo, A.; Algebroid Curves in Positive Characteristics. Lecture Notes in Mathematics 813, Springer (1980).

[4] Decker, W.; Greuel, G.-M.; Pfister, G.; Schönemann, H.; SinguLAR 3-1-1 - A computer algebra system for polynomial computations. http://www.singular.uni-kl.de (2010). 
[5] Drozd Y.A.; Greuel, G.-M.; On Schappert's characterization of strictly unimodal plane curve singularities, In: V.I. Arnold, G.-M. Greuel, J.H.M. Steenbrink:Singularities, The Brieskorn Anniversary Volume. Birkhuser (1998), 326 .

[6] Duc, N. H.; Classification of singularities in positive characteristic, Phd Thesis from University of Kaiserlautern (2012).

[7] Greuel, G.-M.; Lossen, C.; Shustin, E.; Introduction to Singularities and deformations, Math. Monographs, Springer-Verlag (2006).

[8] Greuel, G.-M.; Pfister, G.; A Singular Introduction to Commutative Algebra. Second edition, Springer (2007).

[9] Herzog, J.; Kunz, E.; Die Wertehalbgruppe eines lokalen Rings der Dimension 1, Springer (1971).

[10] Schappert, A.; Kurvensingularitäten und Isomorphieklassen von Moduln,Dissertation, Universität Kaiserslautern (1990).

[11] Zariski, O.; Samuel, P.; Commutative Algebra, Vol. I, II, Springer (1960).

Muhammad Ahsan Binyamin,

Department of Mathematics

GC University, Faisalabad, Pakistan.

Email: ahsanbanyamin@gmail.com

Junaid Alam Khan,

Department of Mathematical Sciences,

Institute of Business Administration,

Karachi, Pakistan

Email: junaidalamkhan@gmail.com

Hasan Mahmood

Department of Mathematics,

GC University, Lahore 54000, Pakistan

Email: hasanmahmood@gcu.edu.pk 